\title{
Numerical response of bridge cranes subjected to repeated shocks during an earthquake
}

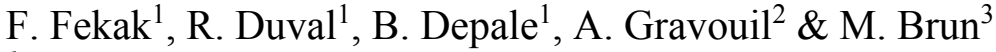 \\ ${ }^{I}$ Pôle Innovation Conception Simulation, France \\ ${ }^{2}$ LaMCoS, Université de Lyon, INSA-Lyon, IUF, France \\ ${ }^{3}$ LGCIE, Université de Lyon, INSA-Lyon, France
}

\begin{abstract}
During an earthquake, a bridge crane may be subjected to multiple impacts between crane wheels and rail. In order to model such phenomena, a time-history dynamic analysis with a multi-scale approach is performed. The high frequency aspect of the impacts between wheels and rails is taken into account by an explicit temporal scheme, modified to resolve impacts. An implicit temporal scheme is used for the rest of the structure. The numerical coupling between the implicit and the explicit schemes is achieved with a heterogeneous asynchronous timeintegrator.

In parallel with the numerical methods, impact tests between crane wheels and different rail mountings were carried out in order to determine a realistic value of the coefficient of restitution used as input for the time-history analysis.

Keywords: earthquake, bridge crane, impacts, heterogeneous asynchronous timeintegrator (HATI).
\end{abstract}

\section{Industrial and scientific context}

\subsection{Bridge crane problematics}

Hoisting appliances such as bridge cranes are heavy mobile systems. They are located overhead in buildings or warehouses.

Usual computational methods of such equipment, subjected to an earthquake, are based on a modal representation of the structures using spectral responses. This method assumes geometric linearity (small deformations and small displacements), material linearity (elastic materials) and a linear behavior at the 
interfaces (no impacts). Consequently, this can lead to an overestimate of seismic loads used to design a bridge crane.

In order to avoid this, it becomes necessary to use sophisticated methods, for instance by introducing vertical and horizontal impacts between crane wheels and rails.

The qualification of these structures with respect to normative seismic design requirements, which are continuously developing, requires strengthened simulation techniques if an overestimation loads used to design this equipment is to be avoid.

Consequently, the use of time-history analysis, based on ground accelerograms, has become more usual.

This non-linear analysis involve more physical phenomena and more numerical parameters. The thesis led by Cetim and LaMCos is aimed at more accurate modelling of the behavior of a bridge crane subjected to earthquake.

Our work follows two stands:

- The development of numerical methods representative of the actual behavior;

- The better understanding of physical phenomena (global and local damping, rebounds, ...).

\subsection{Example of modelling a bridge crane}

A double girder bridge crane is represented in figure 1. Its main structure is composed of two girders, two end-carriages and a trolley.

A bridge crane operation is characterized by three main motions: the trolley (cross travel) motion on the girders, the long travel motion of the crane on the runway rails and the hoisting motion of the lifted load, driven by the hoist installed on the trolley.

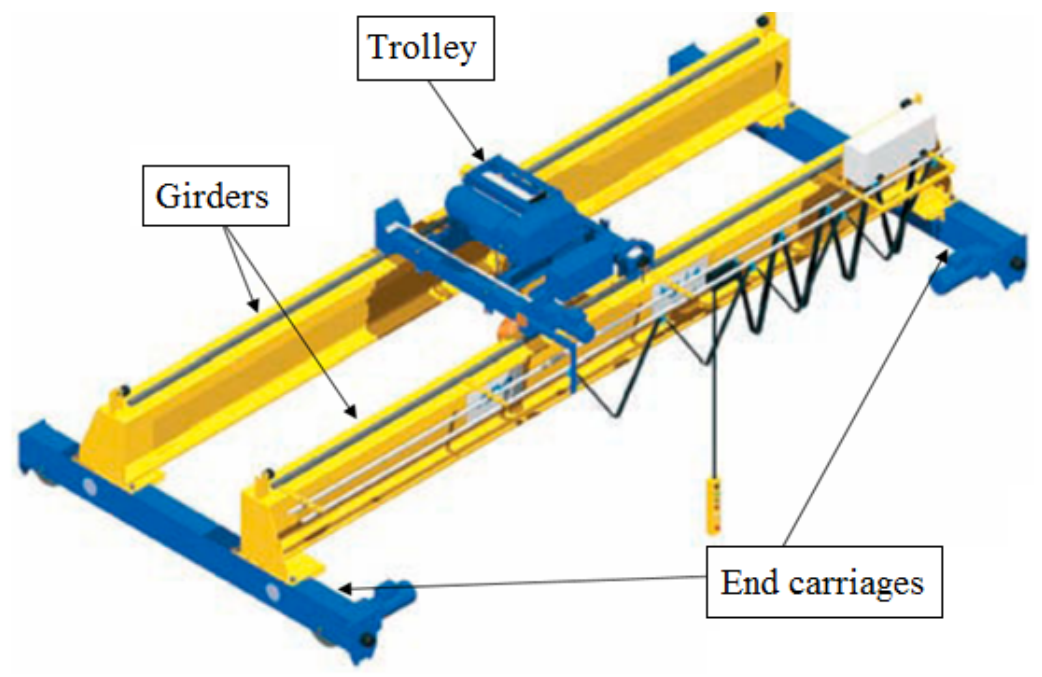

Figure 1: Bridge crane. 


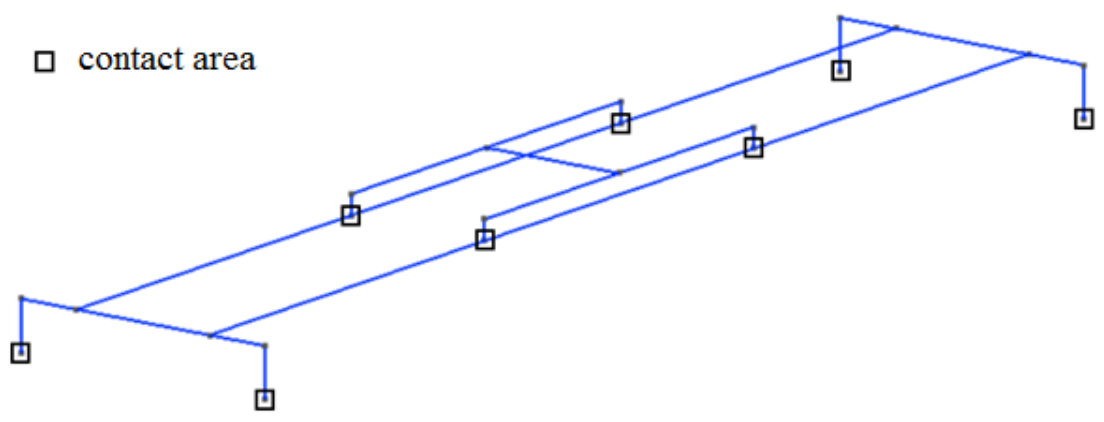

Figure 2: Example of a bridge crane model using beam elements.

The structures of bridge crane are frequently modelled with beam elements, due to the slenderness of the structure components. Figure 2 shows an example of a double girder bridge crane model, marked with the potential contact areas (vertical and horizontal).

\section{Study 1: multi-scale dynamic resolution}

\subsection{Numerical solution strategy}

Because of the impacts to which a bridge crane is exposed during a severe earthquake, several time scales coexist. Therefore, a multi-scale strategy is needs to be adopted. This method allows an adapted time scale to be selected within the subdomain under consideration with better CPU efficiency compared to standard direct monotime step time-integrators.

Consequently, in order to take into account the high frequency characteristics of impacts, we use an explicit temporal scheme in the contact areas. An implicit temporal scheme with greater time steps is adopted for the rest of the structure, for the duration of the seismic excitations.

The coupling is ensured by a heterogeneous asynchronous time-integrators (HATI). Among those coupling methods, is the method of decomposition into subdomains introduced by Gravouil and Combescure [1].

\subsection{Dynamic contact modelling}

\subsubsection{Local formulation in velocity}

We consider a unilateral contact without friction between two bodies $\Omega_{1}$ (wheel) and $\Omega_{2}$ (rail). $\Gamma_{\mathrm{C}}$ is the contact surface.

The unilateral contact formulation, expressed in terms of velocity, is written on the contact surface $\Gamma_{\mathrm{C}}$ as follows (Belytschko et al. [2] and Belytschko and Neal [3]): 


$$
\left\{\begin{array}{l}
\dot{\mathbf{g}}_{\mathrm{N}}=\left(\underline{\mathbf{v}}_{2}-\underline{\mathbf{v}}_{1}\right) \underline{\mathbf{n}}_{1} \geq \mathbf{0} . \\
\mathbf{t}_{\mathrm{N}}^{\alpha}=\underline{\boldsymbol{\sigma}}_{\alpha} \cdot \underline{\mathbf{n}}_{\alpha} \cdot \underline{\mathbf{n}}_{\alpha} \leq \mathbf{0} . \\
\dot{\mathbf{g}}_{\mathrm{N}} \cdot \mathbf{t}_{\mathrm{N}}^{\alpha}=\mathbf{0} .
\end{array}\right.
$$

$\underline{\mathbf{n}}_{\alpha}$ is the external normal to the contact surface $\Gamma_{\mathrm{C}}$, and $\underline{\underline{\boldsymbol{\sigma}}}_{\alpha}$ represents the Cauchy stress tensor existing in the domain $\Omega_{\alpha}$.

The first inequality of eqn (1) represents the non-penetration condition of two solids in contact.

The second inequality of eqn (1) shows that perpendicular loads due to contact can only be compressive. The third equation, also called complementarity condition, reflects that in any time point there is either contact $\left(\dot{\mathbf{g}}_{\mathrm{N}}=0\right)$, or rebound $\left(\mathbf{t}_{\mathrm{N}}=0\right)$.

\subsubsection{Central difference algorithm for a unilateral contact problem}

Based on Lagrange multipliers, the semi-discrete equations of a unilateral contact problem are (Belytschko et al. [2] and Belytschko and Neal [3]):

$$
\left\{\begin{array}{l}
\mathbf{M} \ddot{\mathbf{U}}+\mathbf{F}_{\text {int }}=\mathbf{F}_{\mathrm{ext}}+\mathbf{L}_{C}^{\mathrm{T}} \boldsymbol{\lambda} . \\
\mathbf{L}_{C} \dot{\mathbf{U}} \geq 0 \\
\boldsymbol{\lambda} \leq 0
\end{array}\right.
$$

where: $\mathbf{L}_{\mathrm{C}}$ a rectangular operator which defines the dofs with possible contact, $\boldsymbol{\lambda}$ the vector of the Lagrange multipliers, $\mathbf{M}$ the lumped mass matrix, $\mathbf{F}_{\mathrm{ext}}$ the applied external forces, $\mathbf{F}_{\text {int }}$ the internal forces

To solve the problem (eqn (2)), we use the central difference scheme which is an explicit algorithm. The corresponding equations of this scheme are (Belytschko and Neal [3], Heinstein et al. [4]):

$$
\left\{\begin{array}{l}
\dot{\mathbf{U}}_{n+\frac{1}{2}}=\dot{\mathbf{U}}_{n}+\frac{\Delta \mathrm{t}}{2} \ddot{\mathbf{U}}_{n} . \\
\mathbf{U}_{n+1}=\mathbf{U}_{n}+\Delta \mathrm{t} \dot{\mathbf{U}}_{n}+\frac{\Delta \mathrm{t}^{2}}{2} \ddot{\mathbf{U}}_{n} . \\
\mathbf{L}_{\mathrm{c}, n+1} \dot{\mathbf{U}}_{n+\frac{3}{2}}=0 . \\
\ddot{\mathbf{U}}_{n+1}=\mathbf{M}^{-1}\left(\mathbf{F}_{\mathrm{ext}, n+1}-\mathbf{F}_{\mathrm{int}, n+1}+\mathbf{L}_{\mathrm{c}, n+1} \lambda_{n+\frac{3}{2}}\right) \\
\dot{\mathbf{U}}_{n+1}=\dot{\mathbf{U}}_{n}+\frac{\Delta \mathrm{t}}{2}\left(\ddot{\mathbf{U}}_{n+1}+\ddot{\mathbf{U}}_{n}\right) .
\end{array}\right.
$$


Introducing the mid-step velocity $\dot{\mathbf{U}}_{n+\frac{1}{2}}$ allows the explicit aspect of the central difference scheme to be retained. The contact condition is written at time $(n+3 / 2)$ for solving the equilibrium at time $(n+1)$. It ensures that the contact condition in terms of displacement at time $(\mathrm{n}+1)$ is respected.

\subsubsection{Coupling between explicit and implicit time-integrators}

To couple both numerical schemes (explicit in contact areas and implicit in the rest of the structure), we use the method suggested by Gravouil and Combescure [1].

Therefore, we consider two subdomains $\Omega_{\mathrm{E}}$ and $\Omega_{\mathrm{I}}$, respectively explicit and implicit.

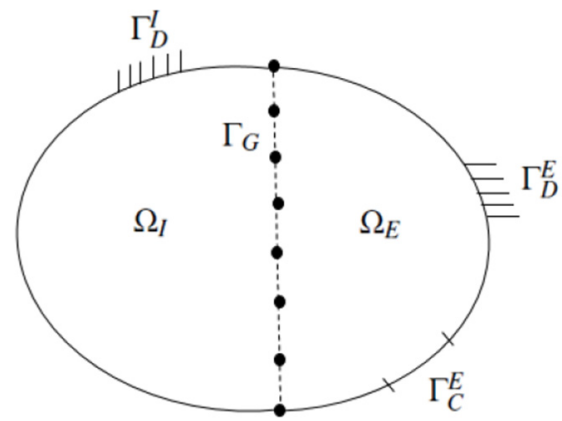

Figure 3: Coupling implicit and explicit subdomains, with contact.

$-\Gamma_{\mathrm{D}}^{\mathrm{E}}$ and $\Gamma_{\mathrm{D}}^{\mathrm{I}}$ are interfaces on which the displacement is imposed.

- $\Gamma_{\mathrm{G}}$ is the coupling interface between both subdomains.

$-\Gamma_{\mathrm{C}}^{\mathrm{E}}$ is the interface of the explicit subdomain where contact occurs.

Let " $\mathrm{j}$ " be the micro-time scale, corresponds to time step of explicit scheme, and " $m$ " be the macro-time scale, corresponds to time step of the implicit scheme. The corresponding equations of the coupling problem, discretized in time and in space, are:

- Equations of equilibrium of each subdomain:

$$
\left\{\begin{array}{rlrl}
\mathbf{M}^{\mathrm{E}} \ddot{\mathbf{U}}_{n+j}^{\mathrm{E}}+\mathbf{F}_{\mathrm{int}, n+j}^{\mathrm{E}} & =\mathbf{F}_{\mathrm{ext}, n+j}^{\mathrm{E}}+\left(\mathbf{L}_{\mathrm{D}}^{\mathrm{E}}\right)^{\mathrm{T}} \boldsymbol{\Lambda}_{\mathrm{D}, n+j}^{\mathrm{E}}+\left(\mathbf{L}_{\mathrm{G}}^{\mathrm{E}}\right)^{\mathrm{T}} \boldsymbol{\Lambda}_{\mathrm{G}, n+j}, \\
& \left(\mathbf{L}_{\mathrm{C}, n+j}^{\mathrm{E}}\right)^{\mathrm{T}} \boldsymbol{\Lambda}_{\mathrm{C}, n+j+\frac{1}{2}}^{\mathrm{E}} & \text { on } \Omega_{\mathrm{E}} \\
\mathbf{M}^{\mathrm{I}} \ddot{\mathbf{U}}_{n+m}^{\mathrm{I}}+\mathbf{K}^{\mathrm{I}} \mathbf{U}_{n+m}^{\mathrm{I}} & =\mathbf{F}_{\mathrm{ext}, n+m}^{\mathrm{I}}+\left(\mathbf{L}_{\mathrm{D}}^{\mathrm{I}}\right)^{\mathrm{T}} \boldsymbol{\Lambda}_{\mathrm{D}, n+m}^{\mathrm{I}}, & \\
& +\left(\mathbf{L}_{\mathrm{G}}^{\mathrm{I}}\right)^{\mathrm{T}} \boldsymbol{\Lambda}_{\mathrm{G}, n+m} . & \text { on } \Omega_{\mathrm{I}}
\end{array}\right.
$$


- Equation at the interface $\Gamma_{\mathrm{G}}$ :

$$
\mathbf{L}_{\mathrm{G}}^{\mathrm{E}} \dot{\mathbf{U}}_{n+j}^{\mathrm{E}}+\mathbf{L}_{\mathrm{G}}^{\mathrm{I}} \dot{\mathbf{U}}_{n+m}^{\mathrm{I}}=0 .
$$

- Equations corresponding to the Dirichlet conditions:

$$
\begin{cases}\mathbf{L}_{\mathrm{D}}^{\mathrm{E}} \dot{\mathbf{U}}_{n+j}^{\mathrm{E}}=0 & \text { on } \Gamma_{\mathrm{D}}^{\mathrm{E}} \\ \mathbf{L}_{\mathrm{D}}^{\mathrm{I}} \dot{\mathbf{U}}_{n+m}^{\mathrm{I}}=0 & \text { on } \Gamma_{\mathrm{D}}^{\mathrm{I}} .\end{cases}
$$

- $\underline{\text { Unilateral contact conditions on }} \Gamma_{\mathrm{D}}^{\mathrm{E}}$ :

$$
\left\{\begin{array}{l}
\mathbf{L}_{\mathrm{C}, n+j}^{\mathrm{E}} \dot{\mathbf{U}}_{n+j+\frac{1}{2}}^{\mathrm{E}} \geq 0 . \\
\boldsymbol{\Lambda}_{\mathrm{C}, n+j+\frac{1}{2}}^{\mathrm{E}} \leq 0
\end{array}\right.
$$

Exponent "E" represents the values linked to the explicit subdomain and exponent "I" represents those of the implicit one. $\mathbf{L}_{G}^{\mathrm{i}}, \mathbf{L}_{\mathrm{C}}^{\mathrm{i}}, \mathbf{L}_{\mathrm{D}}^{\mathrm{i}} ; \mathrm{i}=\mathrm{E}$, I are the matrices respectively corresponding to the relations at the interface $\Gamma_{\mathrm{G}}$, the contact condition at interface $\Gamma_{\mathrm{C}}$ and the Dirichlet conditions on $\Gamma_{\mathrm{D}}^{\mathrm{i}} ; \mathrm{i}=\mathrm{E}$, I. The $\boldsymbol{\Lambda}_{\mathrm{j}}^{\mathrm{i}}$; $\mathrm{i}=\mathrm{E}, \mathrm{I}$ and $\mathrm{j}=\mathrm{D}, \mathrm{C}, \mathrm{G}$ are the matrices of the Lagrange multipliers.

\section{Study 2: time-history analysis, improved by tests}

\subsection{Wheel/rail impact tests with different mountings}

In parallel with the multi-scale dynamic resolution method mentioned above, Cetim has investigated the local aspect of a wheel/rail impact.

In time-history analysis of bridge cranes, impact parameters are not well known, by lack of experimental results, vertical shocks are top priority.

This is why Cetim has experimentally studied the coefficient of restitution of impacts between rails and wheels. This coefficient of restitution is defined as the ratio of speeds after and before an impact. This coefficient is linked to the dissipation of energy during the contact.

The final purpose is to be able to model the rebounds and their dissipation in dynamic non-linear calculation models.

Those tests were performed in the VALUTEC Test center of the University of Valenciennes, with an impact drop tester.

A vertical mobile trolley (wheel) is dropped on a rail (see fig. 4 below). Falls heights vary from 15 to $452 \mathrm{~mm}$ and weight from $83 \mathrm{~kg}$ to $128 \mathrm{~kg}$. 


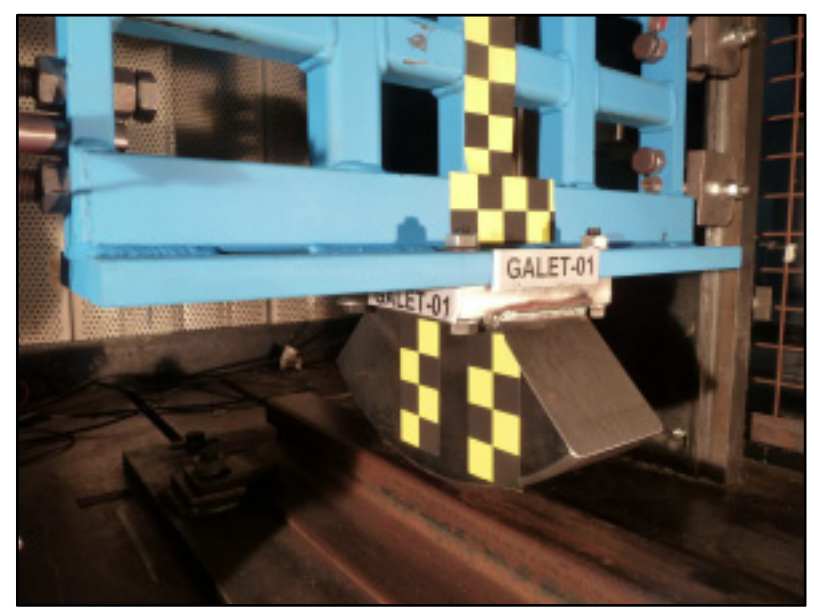

Figure 4: Test bench.
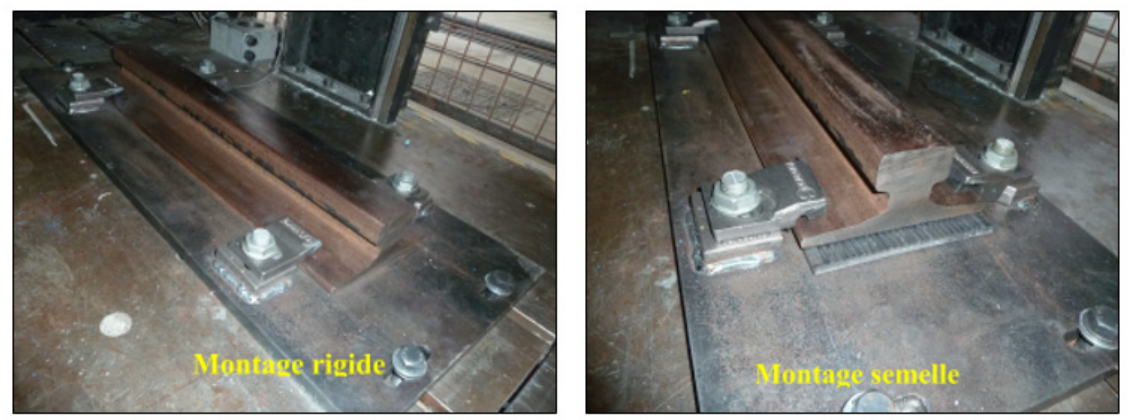

Figure 5: Rigid and elastic mountings of rails.

Two rail mountings were investigated (see fig. 5):

-A simple rail fixed by adjusting clips: rigid mounting;

-A rail mounted on a resilient sleeper-pad: elastic mounting.

Thanks to those tests, a range of values could be found for the coefficient of restitution.

\subsection{Local contact model}

After those tests, a further step has consisted in developing a local element allowing to dissipate a chosen quantity of energy, for a wheel/rail contact under consideration.

This local element could be developed and tested with ANSYS elements, it is composed of three basic elements (see Table 1 below). 
310 Earthquake Resistant Engineering Structures X

Table 1: Local element.

\begin{tabular}{|l|l|l|l|}
\hline A spring & $\begin{array}{l}\text { A normal } \\
\text { contact } \\
\text { (vertical) }\end{array}$ & A dissipating system \\
\hline & & & \\
\hline
\end{tabular}

The current step of the study consists in the modelling of the bridge crane using the local elements mentioned above. 
Different versions of the model are studied, with an increasing difficulty:

-Model n¹:

Linear spectral analysis of the bridge crane, the response spectrum being generated from the accelerograms of the time-history analysis.

\section{-Model n'2:}

Time-history analysis with a global damping (Rayleigh type). Normal contacts will be modelled by unilateral contacts linked to a contact stiffness. Only one set of data (simultaned accelerations along $\mathrm{X}, \mathrm{Y}$ and $\mathrm{Z}$ axis).

\section{-Model n³:}

Model $\mathrm{n}^{\circ} 3$ with addition of the local elements defined figure 5 , with the same global Rayleigh type damping.

\section{-Model n4:}

Model $\mathrm{n}^{\circ} 3$ without the massic term in the Rayleigh type damping.

\section{-Model n'5:}

Model $\mathrm{n}^{\circ} 3$ without Rayleigh type damping. Only contacts (normal dissipation and friction) will dissipate energy.

\section{Conclusion and outlooks}

Time-history dynamic analysis with a multi-scale approach allows accurate numerical resolution. Each subdomain can have its own temporal integrator (heterogeneous) and its own time scale (asynchronous) (Gravouil et al. [6]).

The development of such algorithm will make it possible in a second step to gain significantly CPU time in the three-dimensional modelling of bridge cranes subjected to earthquakes.

Exploring physical phenomena, impact tests between wheels and rails have resulted in realistic values of the coefficient of restitution, allowing optimized time-history analysis with FE packages such as ANSYS or ABAQUS. Coefficient of restitution being not available in such software, a local model based on friction has been developed. This local model allows input of a coefficient of restitution in time-history analysis.

Both works follow a different topic but are aimed to converge towards the same goals; being accuracy, robustness and easy-to-use.

\section{References}

[1] Gravouil, A., Combescure, A., A multi-time-step explicit-implicit method for non-linear structural dynamics, IJNME, 50, pp. 199-225, 2001.

[2] Belytschko, T., Liu, W.K. \& Moran, B., Nonlinear Finite Elements for Continua and Structures, John Wiley and Sons, Ltd., 2000.

[3] Belytschko, T., Neal, M.O. Contact-Impact by the Pinball Algorithm with Penalty and Lagrangian Methods. IJNME, 31, pp. 547-572, 1991.

[4] Heinstein, M.W., Mello, F.J., Attaway, S.W. \& Laursen, T.A. Contact-Impact Modeling in Explicit Transient Dynamics, CMAME, 187, pp. 621-640, 2000. 
312 Earthquake Resistant Engineering Structures X

[5] Casadei, F., A Hierarchic Pinball Method for Contact-Impact in Fast Transient Dynamics. Conf. VI Congresso Nazionale della Società Italiana di Matematica Applicata e Industriale (SIMAI), Italy, 2002.

[6] Gravouil, A., Combescure, A. \& Brun, M., Heterogeneous asynchronous time integrators for computational structural dynamics, IJNME, accepted, 2014. 